\title{
From Fear To Fun In Thermodynamics
}

\author{
David Roundy*, Ayush Gupta†, Joseph F. Wagner", Tevian Dray*, Mary Bridget \\ Kustusch* and Corinne A. Manogue*
}

\author{
*Department of Physics, Oregon State University, 1500 SW Jefferson Way, Corvallis, OR 97331 \\ † Department of Physics, University of Maryland, 8082 Baltimore Ave, College Park, MD 20740 \\ "I Department of Math, Xavier University, 3800 Victoria Parkway, Cincinnati, OH 45207
}

\begin{abstract}
The complexity of thermodynamics challenges many students as well as faculty. Understanding what a partial derivative represents may be key to reducing the anxiety associated with this topic. In this session, participants engaged with a sequence of activities designed to elucidate the mathematics of thermodynamics through multiple representations of partial derivatives. Activities include: experiments that provide exemplars of measuring thermodynamic quantities involving partial derivatives, thought experiments where students design ways to measure particular partial derivatives representing thermodynamic quantities, a mechanical analogue that physically represents changes that hold specific quantities fixed, and an algebraic formulation of a partial derivative chain rule. Our discussants, Ayush Gupta and Joseph Wagner, each comment on how their different research perspectives can contribute to and are necessary for a holistic understanding of what happens during this kind of curricular sequence.
\end{abstract}

Keywords: thermodynamics, partial derivatives, experiments PACS: $01.40 . \mathrm{Di}, 01.50 . \mathrm{Qb}$

\section{INTRODUCTION}

Many students (and faculty!) find upper-division thermodynamics challenging and confusingespecially in terms of its idiosyncratic mathematics [15]. Yet we love physics because it enables us to understand the world around us using mathematics. As part of the Paradigms in Physics Project, we have developed a sequence of activities that bring fun back into thermodynamics. Five activities in our Energy and Entropy course help students to connect thermodynamic and mathematical concepts, especially partial derivatives, with concrete reality.

In this 2013 PERC session, attendees had a handson opportunity to try out these activities. This paper begins with a brief description of each activity. The reader will find complete instructions for setting up (easy) and using these activities in the classroom on the Paradigms website [6] as well as further discussion in the papers cited below. This paper will conclude with a summary of how the session's two discussants (AG and JFW) saw these activities through the lenses of their separate research perspectives.

\section{Ice Calorimetry}

Our students' first experience in Energy and Entropy is a laboratory experiment measuring the latent heat of fusion of ice and the heat capacity of water. We give students a resistive heater, a styrofoam cup, a scale, a multimeter, a thermometer, and some ice (a)

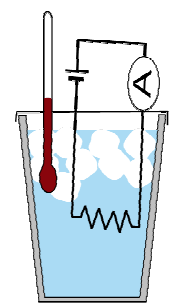

(b)

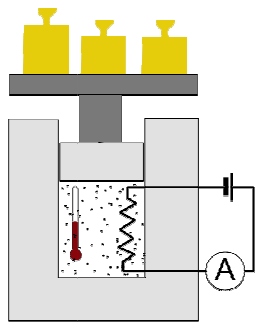

FIGURE 1: (a) Sketch of the ice calorimetry experiment performed on the first day of class. (b) Solution to a "namethe-experiment" activity in which students are asked to imagine an experiment measuring $(\partial S / \partial T)_{p}$. Students commonly point out the connection in the second name-theexperiment activity that this experiment is the same as they did in the first week of class.

and water, and ask them to measure how much energy is required to first melt the ice and then to warm up the water. This experiment is sketched in Fig. 1. The energy is measured with freshman physics concepts, using the multimeter. Students find the latent heat of melting and specific heat of water, using measurements of the energy required to melt the ice and the slope of the temperature with respect to energy added.

Students further analyze their data to find the change in entropy of the system during this heating process, using the thermodynamic definition of entropy

$$
\Delta S=\frac{\int-\bar{d} Q_{\text {quasistatic }}}{T}
$$

where $-\bar{d} Q$ is a small amount of energy added to the system by the heater, and the quasistatic subscript is a reminder that the ice water needs to be thoroughly 
stirred as the heating is performed. This experiment gives our students their first exposure to entropy in our course.

\section{Rubber bands}

After we introduce Maxwell relations, midway through the course, we do an experiment. Students measure the change in internal energy and entropy when a rubber band is isothermally stretched, using the Maxwell relation derived from the Helmholtz free energy, which is

$$
\left(\frac{\partial S}{\partial L}\right)_{T}=-\left(\frac{\partial \tau}{\partial T}\right)_{L}
$$

where $\tau$ is the tension in the rubber band, and $L$ is its length. This relation connects a derivative of the tension with respect to temperature with a derivative of the entropy with respect to length. This allows students to numerically integrate to find the change in entropy for a finite isothermal stretch. This experiment requires students to experimentally evaluate partial derivatives and to integrate experimentally measured quantities. This reinforces the idea that these mathematical manipulations describe concrete physical reality.

For more detail, see the recently published paper describing this activity [7].

\section{Name the Experiment}

Three times during the course we have "name-theexperiment" activities. We ask student groups to describe and sketch an experiment that would measure a given thermodynamic partial derivative [8]. Namethe-experiment activities require students to make use of operational definitions of thermodynamic quantities, articulate how they would hold a given variable constant, and (in the later activities) perform manipulations on derivatives in order to find a quantity that is easier to measure.

These activities work synergistically with the laboratory experiments described above. Students often recognize that they have sketched an experiment similar to one that they actually performed. As an example, consider the derivative $(\partial S / \partial T)_{p}$. This derivative is closely related to the heat capacity, which students measure in the ice calorimetry lab. A namethe-experiment solution is shown in Fig. 1, next to the diagram of the experiment they performed.

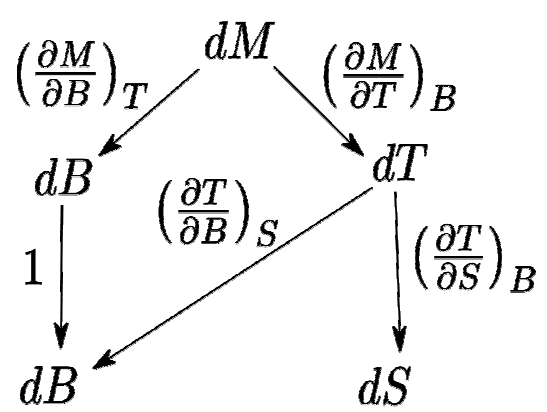

FIGURE 2: A chain rule diagram using differentials.

\section{Partial Derivative Machine}

This year we added a new week of hands-on activities that precede the Energy and Entropy course, which are intended to teach students the mathematics they will need in order to work with differentials and partial derivatives. These activities (discussed in more detail in two separate papers in these Proceedings [9, 10]) use a mechanical analogue of thermodynamics, in which two distances and two forces play the roles of entropy, volume, temperature, and pressure. This allows students to grapple with partial derivatives (both literally and figuratively) in a field of physics that is comfortable. We use this elastic system to illustrate and teach everything from partial derivatives, total differentials, the thermodynamic identity (except that it is not thermodynamic), Maxwell relations and Legendre transforms.

\section{Chain Rule Diagrams}

Many homework and exam problems in thermodynamics expect students to be fluent in manipulating partial derivatives through the repeated application of various chain rules. The common experience of becoming totally lost in this forest of equations contributes to students' fear of this subject. Partial derivative diagrams are common to many multivariable calculus texts $[11,12]$. We have adapted partial derivative diagrams to the language of differentials-which are anathema to many mathematicians. The reader can try using the chain rule diagram in Fig. 2 as a map to find the adiabatic magnetic susceptibility

$$
\chi_{S}=\left(\frac{\partial M}{\partial B}\right)_{S}
$$

for a system, given complicated expressions for $M$ and $S$ in terms of $B$ and $T$-sufficiently complicated that it is impossible to solve for $M(B, S)$. Add together the contributions from all possible paths on the map from $d M$ to $d B$, and multiply together all the partial 
derivatives that lie on a given path. More information on this strategy can be found in [13-15].

\section{DISCUSSANTS}

\section{Ayush Gupta}

My first reaction to the activities was one of curiosity, playful confusion, and a call to sensemaking. I felt drawn to start manipulating the partial derivative machine even before I was briefed formally on how the machine is supposed to work. Similarly, coming up to the "rubber-bands experimental set-up" naturally raised the question for me: Will the tension in the chain (connected to the rubber band) increase or decrease as the water is heated up? The set-up invites one to start asking interesting inquiry questions. So the developed activities can open up pathways for students to start approaching the "feared" ideas of thermodynamics in a more playful manner.

It is not clear to me whether my experience is typical of how the students experience these activities. While mine and the other authors' intuition says that these activities should ease some of the fears traditionally associated with thermodynamics and help facilitate learning in that domain, I think that future work should aim to address (1) whether the activities have the intended effect (assessment) and (2) how the activities alleviate fear or facilitate learning (mechanism). This raises the following question: if a designed curriculum achieves the intended curricular goals, then why should we undertake the extensive work of showing how it works? I have multiple reasons to argue that it is essential that we undertake that effort.

For one, systematic research into how students approach learning in the context of these novel activities can help us refine the curriculum and its implementation [16, 17]. For example, while it was challenging and fun for me to play with arguments and counter-arguments for why the tension in the chain might increase as the rubber band is heated up, the focus of the analysis of the experiment is on the mathematics of entropy change as the rubber band is stretched-a much more structured activity. As a workshop participant, I felt I had the freedom to explore the question that came to my mind when I saw the set-up. As a student, would I have felt the same freedom to explore or would I have felt compelled to simply do what I was being told to do? What might the affective and conceptual consequences be for such a student? What role do the epistemological messages embedded in the worksheet play in constraining how students approach the task? How students approach a task can significantly influence what knowledge resources they bring to bear and whether they are successful or not $[18,19]$. Of course, with a diversity of students, there will be a diversity of ways in which they experience the activities. But my point is that exploring how students approach and experience the activities can help us better tailor the design and implementation of curriculum.

Second, instructors are known to adapt rather than adopt curricular materials. So a more fleshed-out knowledge-base on how students approach these activities can help instructors adapt the activities to fit their instructional goals in a more informed manner.

Finally, developing this knowledge-base can add fundamental knowledge to our understanding of how students approach learning physics and mathematics in the context of these novel activities that draw on students' formal (physics, mathematics), informal (everyday sense-making), and kinesthetic knowledge. For example, recent work by Sian Beilock and Susan Fischer shows that students learn concepts such as angular momentum better if they are made to have sensorimotor experiences related to the concept [20]. Beilock and Fischer pose neurological mechanisms for why this might be the case: that the kinesthetic experience engages the motor system of the brain, which is also shown to be active when students later think about the concept without the physical stimulation. Does engaging students in kinesthetic experiences that involve partial derivatives work through a similar mechanism? Or does learning partial derivatives via the partial derivative machine work through a different mechanism? This knowledge-base can further spur the development of and research on such activities, more than if the community had access only to the designed activities.

\section{Joseph F. Wagner}

A particular appeal of some of these activities is their ability to engage students simultaneously in making sense of physics and making sense of mathematics. Many of the mathematical ideas used within these activities (e.g., partial derivatives, numerical integration) are known to students primarily or exclusively in the context of static, spatial/geometric interpretations typically used in mathematics classrooms. In the context of these activities, however, students are expected to apply their mathematical knowledge to model dynamically changing phenomena that they can experience, measure, and sometimes even feel kinesthetically.

Traditional investigations of knowledge transfer ask how it is that something learned in one context is later used and applied in a new and different context. At face value, this would suggest that students need to take knowledge of mathematics that they already know 
and "apply" it in the context of these activities. We know, of course, that students struggle to transfer their mathematical knowledge to these activities [7], quite possibly because of the mismatch between the static, geometric explanations and representations used in mathematics classrooms, and the dynamic and often non-spatial phenomena they need to model. This suggests that what occurs with students engaging in these activities is not simply an application of previously learned mathematical knowledge, but a refinement and development of that knowledge that is, in fact, transformed by their reasoning supported by the affordances of the new contexts.

As an example, it is common for students to complete their calculus courses with an understanding of integration as a means of finding "area under a curve." Even though more dynamic interpretations of integration as an accumulation of a changing quantity may receive some attention, the dominance of the spatial representations in mathematics classrooms maintains the "area under the curve" interpretation as primary, even exclusive. Only with the experience of new contexts, particularly contexts involving dynamic and non-spatial phenomena, are students able to develop a more broadly applicable understanding of integration as accumulation. From this perspective, the typical geometric and spatial representations and interpretations of central ideas in calculus should not be thought of as abstract (as I believe mathematicians tend to think of them), but rather as very particular contextual instantiations of mathematical ideas whose complexity cannot be adequately conveyed in such a limited context.

I believe that activities such as those presented here offer opportunities for educational researchers in both physics and mathematics interested in student learning and cognition, especially for researchers interested in knowledge transfer. Consistent with my earlier work [21], I propose that students engaging in activities such as these do not transfer their existing knowledge into a new context but, rather, that their experiences in the new contexts challenge students to transform and refine their existing knowledge. As a result, we may expect to see as the fruit of these activities the simultaneous development of students' knowledge of physics and mathematics. At least, this is my theory. If supporting evidence is to be found, these activities seem an ideal place to look for it.

\section{CONCLUSION}

We have presented a sequence of activities that ground thermodynamics in tangible, physical reality for students. Anecdotal evidence suggests that these activities contribute to removing the fear from thermodynamics

\section{ACKNOWLEDGMENTS}

The organizers of this session would like to thank our two discussants, Ayush and Joe, for bravely taking on the task of publicly trying to fit the work of others into their own framework. We greatly appeciate the insights they have shared with us. We also thank the members of the Paradigms team who helped with the workshop: Novela Auparay, Len Cerny, Grant Sherer, and Justyna Zwolak.

\section{REFERENCES}

1. A. R. de Ataìde, and I. M. Greca, Science \& Education 22, pp. 1405-1421 (2012).

2. B. Bucy, J. Thompson, and D. Mountcastle, PERC Proceedings pp. 157-160 (2006).

3. E. Pollock, J. Thompson, and D. Mountcastle, PERC Proceedings pp. 168-171 (2007)

4. J. R. Thompson, B. R. Bucy, and D. B. Mountcastle, PERC Proceedings pp. 77-80 (2005).

5. J. R. Thompson, C. A. Manogue, D. J. Roundy, and D. B. Mountcastle, PERC Proceedings pp. 85-88 (2011).

6. Paradigms in Physics project website, http://physics.oregonstate.edu/portfolioswiki (20072013).

7. D. Roundy, and M. Rogers, Am. J. Phys. 81, 20 (2013).

8. D. Roundy, M. B. Kustusch, and C. Manogue, Am. J. Phys. (2013), in press.

9. G. Scherer, M. B. Kustusch, C. Manogue, and D. Roundy, PERC Proceedings (2013), in press.

10. C. A. Manogue, E. Gire, and D. Roundy, PERC Proceedings (2013), in press.

11. D. Hughes Hallett, W. McCallum, et al., Calculus, Wiley, 2012, 6th ed.

12. J. Stewart, Calculus, Brooks/Cole, 2012, 7th edn.

13. T. Dray, M. B. Kustusch, C. A. Manogue, and D. Roundy, Working with Chain Rule Diagrams (2013), in press.

14. T. Dray, and C. Manogue, The Geometry of Vector Calculus, in press.

15. D. Roundy, T. Dray, M. B. Kustusch, E. van Zee, and C. A. Manogue, From Fear to Fun in Thermodynamics session, in press.

16. E. F. Redish, and R. N. Steinberg, Physics Today 52, 2431 (1999).

17. L. C. McDermott, Am. J. Phys. 69, 1127 (2001).

18. L. Lising, and A. Elby, Am. J. Phys. 73, 372 (2005).

19. A. Gupta, and A. Elby, International Journal of Science Education 33, 2463-2488 (2011).

20. S. Beilock, and S. Fischer, PERC Proceedings (2013), in press.

21 J. F. Wagner, Cognition and Instruction 24, 1-71 (2006). 